Ann. Sci. forcst., $1976,33(4), 221-245$.

\title{
L'INFLUENCE DES REBOISEMENTS SUR LES COMMUNAUTÉS D'OISEAUX L'EXEMPLE DU MONT VENTOUX (')
}

\author{
J. BLONDEL \\ Laboratoire d'Ecologic et de Phytosociologie. \\ Université des Sciences at Techniques du Languedoc, \\ 5, ne Auguste Broussonet, \\ 34000 Montpellier
}

RÉSUMÉ

Un diagnostic écologique des communautés d'oiseaux a été réalisé dans douze formations forestières spontanées et artificielles du Mont Ventoux. L’analyse des résultats a révélé que :

- $5 \mathrm{r}$ espèces d'oiseaux soit $6 \mathrm{r}$ p. roo de l'avifaune nicheuse totale du massif ont été recensées dans les forêts. L'examen de la répartition des espèces dans les différentes formations montre que les reboisements ont a reconstruit $\times$ une avifaune forestière qui n'est pas fondamentalement différente de celle des forêts spontanées;

- le milieu le plus riche et le plus diversifié est la Cédraic. En moyenne, les forèts du Ventoux, qu'elles soient spontanées on artificielles contiennent des communautés d'oiseaux variées et equilibrées (tabl. 4) :

- la richesse et la diversité des peuplements d'oiseaux sont étroitement fonction de la diversité de structure de lit végétation (fig. 1). Les oiseaux réagissent de la même façon à ce paramètre, que la formation soit spontanéc on artificiclle :

- plus le paysage forestier est varié, plus la communauté contient des espèces rares et exigentes dans le choix de leur habitat ( - faible largeur de niche). Dans les monocultures équiennes de conifères dont la structure de la végétation est très simplifiée, on ne trouve en revanche que quelques espèces banales et uhiquistes:

- les coefficients de ressemblance entre les différentes communautés sont élevés et ne permettent pas d'isoler les reboisements des formations spontanécs. Huit formations dont cinq sont spontanées (Hêtraie-Sapinière, Pineraie de Pins sylvestres, Pineraie spontanée de Pins à crochets, boisements mixtes de Chểnes pubescents $\times$ Pins sylvestres et chènaie de Chênes pubescents) et trois artificielles (Pineraie de Pins noirs, Cédraie, Pineraie artificielle de Pins à crochets) forment un ensemble homogène relié au niveau de similitude de 0,75 qui est très élevé.

On en conclut que les formations sylvatiques naturelles et artificielles du Ventoux contiennent des communautés riches et variées dont beaucoup ont été indirectement "reconstituées *

(1) Ce travail a été subventionné par la Délégation générale à la Recherche scientifique et technique, contrat $\mathrm{n}^{\circ} 74-70-100$ et par le Centre national de la Recherche scientifique, Action thematigue programmé - Dynamique des Populations ", Décision n* 1893. 11 a été réalisé avec l'amicale collaboration de Paul du MExt.: animateur de r'Action concertée D.G. K.S.T. + Équilibres et Lutte biologique in dans le Ventoux. 
par l'homme. On discute ensuite quelques mécanismes de l'occupation de l'espacs par les oiseaux en s'appuyant sur le cas du milieu le phus riehe, la Cédraie et sur celue da plus pauvre, la Pineraie artificielle de Pins à crochets. L'argumentation permet de conclure que le succès das aménagements forestiers est d'autant meilleur qu'il a pour effet d'augmenter la diversité de strusture de la végétation (traitement en futaie jardinée) ainsi que celle du cortège floristig̨ue spontane.

On souligne entin la différence fondamentale qui existo entre fospls de production ct forits de protection, ce qui amène nécessairement à insister sur le fait que les résultats exposts ne sont valables que dans le cas specifique tu Vientous.

\section{I. - INTRODUCTION}

Fin I959. FERRy avait attiré Pattention des biologistes et forestiers sur les modifications que l'action du sylviculteur apporte aux populations d'oiseaux. Pour la première fois en France dans ce domaine, la mesure objective se substituait à l'intuition et ouvrait la voie à l'exploration scientifique chiffrée de l'impact des aménagements forestiers sur les avifaunes naturelles. Cette ouvre de précurseur allait susciter un très grand nombre de travaux portant sur l'affinement des méthodes et sur l'étude approfondie de la composition et de la dynamique des peuplements d'oiseaux en fonction de la structure de la forèt et des modifications qu' elle subit le long des révolutions forestières (cf. FERRY et Frochot, r970, 1974: BLONDEI, et al., 1973). Mis à part quelques travaux plus ou moins détaillés (YArP, I962;

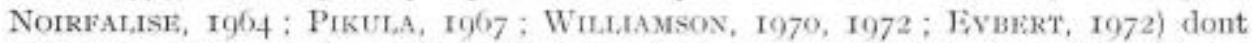
aucun ne se rapporte à la région méditerranéenne, nous manquions toutefois d'études précises sur l'incidence des reboisements, notamment des enrésinements, sur l'avifaune sylvatique. Or, de tous les aménagements forestiers, ce sont probablement ces derniers qui ont été l'objet ces dernières années des discussions les plus animées entre les naturalistes et les gestionnaires de nos forcts. Il nous a donc paru intéreseant. d'étudier cette question dans cet admirable champ d'expérience que constitue le Mont Ventoux grâce à un protocole rigoureux d'échantillonnage et d'analyse que nous venons précisément de mettre au point dans le dessein de formuler un diagnosfic écologique de l'suironnement à partir des communautés d'oiseaux (BLoNDis, I975).

Le Mont Ventoux est un puissant anticlinal construit dans les couches calcaires du Crétacé inférieur et orienté d'ouest en est. Le pli, très dissymétrique, présente un versant nord beaucoup plus redressé que le versant sud dont la pente régulière (20 p. roo en moyenne) présente des conditions particulièrement favorables à l'étude de l'étagement de la végétation: " Le Ventoux, par la succession remarquable de ses étages et séries de végétation, peut étre considéré comme la montagne la plus représentative des Préalpes du Sud, et ce, malgré la dégradation de certaines de ces séries n (BARbero et Quiziñ, I975). Ce versant qui présente en outre l'intérêt de contenir les plus beaux massifs de reboisement a constitué depuis plusieurs années le théatre de nos observations car nous nous trouvions dans une situation particulièrement favorable oì nous pouvions comparer les peuplements d'oiseaux des reboisements à ceux des formations spontanées. Ces forêt. qui appartiennent à la commune de Bédoin depuis que le Seigneur des Baux lui en a fait don au xmt siècle 
se développent sur quelque 6300 ha entre le Piémont (400 m) et le sommet ( $\mathrm{I}$ 9I2 m) et sont soumises au Régime forestier.

Comme chacun sait, le Ventoux est, avec 1'Aigoual (cf. NÈGre, I931) 1'un des plus illustres exemples français de reconstitution d'une couverture forestière digne de ce nom après que la hache, les essarts, le feu et les excès du pâturage en aient supprimé la plus grande partie. Au milieu du siècle dernier, la moitié inférieure du versant sud n'était qu'un $\alpha$ désert de pierres * (MAURY, I 960 ). Ce fut la $\alpha$ région du Thym et des Lavandes complètement dépourvue d'arbres * (MAR'rrss, I838) qui témoigne de l'éradication de la forêt entre les cotes $550 \mathrm{~m}$ et I $\mathrm{I} 50 \mathrm{~m}$ comme on peut le voir sur la coupe saisissante publiée par cet auteur et reproduite dans les travaux de MAURy (loc, cit.) et de Gcisrt et Pautou ( 1969$)$ entre autres. Après de multiples cris d'alarme dont les premiers furent vainement lancés dès $1_{549}$, deux dates marquantes jalonnent l'histoire de la forêt de Bédoin : la soumission au Régime forestier de 4473 ha le 3 juin I830 et surtout le décret du 22 mars I86r qui lança les opérations de reboisement dans le dessein d'enrayer la disparition des sols et dont 1'historique est retracée par Maury (I960).

Le plus gros de l'effort eut lieu entre $186 \mathrm{r}$ et 1873 où l'on reboisa 2500 ha mais les opérations se poursuivirent à un rythme variable jusqu'à la Seconde Guerre mondiale; de nouveaux programmes sont actuellement en cours d'élaboration. Comme le souligne MAURY (loc. cit.) les travaux furent entrepris avec un bon sens, une opiniâtreté et un souci d'économie et d'efficacité qui forcent notre admiration et que récompensèrent largement les magnifiques résultats dont nous bénéficions aujoutd'hui.

A chaque étage de végétation ont été choisies avec discernement les essences les plus aptes à donner de bons résultats : du Chêne vert et du Pin d'Alep aux étages inférieurs au Pin à crochets dans les zones les plus élevées (tabl. I). Soulignons au passage la sagacité de ces reboiseurs qui n'ont pas hésité à remettre à leur place les Feuillus détruits par l'homme : Chêne vert ( 3000 ha), Chêne pubescent dont Maririss (1838) disait qu'il était " presque éliminé " et même le Hêtre.

Bref, de Bédoin au sommet du Ventoux, lorsque le temps est clair, l'observateur peut embrasser du regard ce manteau forestier aujourd'hui rétabli et distinguer les différents massifs à la couleur d'ensemble que confèrent au paysage les essences dominantes qui se relaient du $\Gamma$ ém ont à la ceinture subsommitale de Pins à crochets. L'étagement des formations forestières est analogue sur le versant nord mais la raideur de la pente qui téléscope les étages et la masse dominante des Pins noirs d'Autriche rendent le spectácle moins saisissant.

Si l'ampletur des reboisements complique la tâche du Botaniste désireux de reconstituer les séries de végétation spontanées (BARBrko et al., 1976), il faut reconnaître que pour les études que nous avons menées, la coexistence de forêts spontanées et de vieux reboisements nous plaçait dans une situation quasi expérimentale faisant du Ventoux ce * laboratoire naturel de choix * (BARBEro et al., loc. cit.) auquel rêve le naturaliste.

La végétation naturelle du Ventoux, très étudiée, a récemment été l'objet de plusieurs synthèses dont il nous suffira ici de citer celle de BARBEro et al. (I976) réalisée dans le cadre du même contrat * Êquilibres et lutte biologiques * et à laquelle le lecteur se reportera utilement pour plus amples renseignements. Ces auteurs reconnaissent 8 séries de végétation que se partagent un * complexe méditerranéen * 
et un « complexe médioeuropéen ^. Les séries sylvatiques et leurs faciès de dégradation peuvent être regroupés en 5 étages. Nous nous contenterons pour les besoins de notre argumentation d'indiquer au regard de ces étages les principales formations spontanées qui s'y rapportent ainsi que les reboisements qui ont été effectués (tabl. I).

\section{TABI,EAU I}

Etagement schimatique des principales formations forestières et des reboisemonts ais Mont Veutuax

(situation en adret sauf pour la Hètraie-Sapinière)

\begin{tabular}{|c|c|c|c|c|}
\hline $\begin{array}{l}\text { Êtages de végétation } \\
\text { (sensu BARnE ro et af.) }\end{array}$ & Formations spotitanices & Reboisen: & & $\begin{array}{l}\text { Repéres altitudinaux } \\
\text { (mètres) }\end{array}$ \\
\hline $\begin{array}{l}\text { Méditerranéen } \\
\text { Supraméditerranćen } \\
\text { Moutagnard-méditerran. } \\
\text { Oroméditerranéen }\end{array}$ & 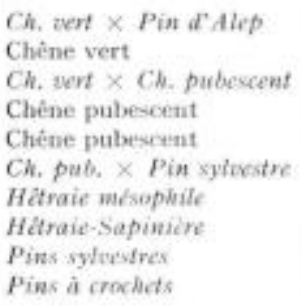 & $\begin{array}{ccc}\hat{c V} & & \hat{\imath} \\
\downarrow & & P \mathrm{MI} \\
& \uparrow & \downarrow \\
& \hat{C} & \hat{\imath} \\
\hat{i} & \downarrow & P \mathrm{~V} \\
\downarrow & & \downarrow\end{array}$ & $\begin{array}{c}\uparrow \\
P A \\
\downarrow \\
\uparrow \\
C P \\
\downarrow \\
\uparrow \\
P C \\
\downarrow\end{array}$ & $\begin{array}{l}500-500 \\
550-600 \\
500-700 \\
600-900 \\
800-1500 \\
900-1500 \\
1200-1500 \\
1200-1500 \\
1500-1600 \\
+500-1800\end{array}$ \\
\hline
\end{tabular}

Nâes : 1. La dénomination des formations ne correspond pas nécescairement aux séries climaciogues définies par BakBz uo of al, (1976).

2. Les formations dont le nom est en italiques ont eté l'objet d'une diagnose ornithologique. $\mathrm{CV}=$ Chéne vert $; \mathrm{PA}=\mathrm{Pin}$ d'Alep; $\mathrm{PXI}=$ Pin maritime ; $\mathrm{CP}=$ Chène pubescent ; $\mathrm{C}-$ Cedre ; PN - Pin noir d'Autriche; $\mathrm{PS}=$ Pin sylvestre; PC = Pin à crochets.

A ces reboisements entrepris sur une grande échelle, rappelons pour mémoire que d'autres essences ont été plantées plus ou moins à titre d'essai : 1'Épicéa notamment ainsi que quelques Mélèzes et Pins cembro. Bien que dans certains cas, il soit difficile aujourd'hui de délimiter franchement les formations spontanées des reboisements, notamment pour certaines Chênaies et Pineraies de Pins d'Alep, 3 des 12 formations étudiées sont des résineux entièrement artificiels (Cédraie, Pineraie de Pins noirs, Pineraie de Pins à crochets), 5 sont spontanées ou subspontanées (Hètraie-Sapinière, Hêtraie mésophile, Pineraie de Pins à crochets, Pineraie de Pins sylvestres, bois mixtes de Chênes pubescents et Pins sylvestres, ces derniers étant d'ailleurs des faciès à Pins sylvestres de dégradation de la Chênaie pubescente, BARBERo, in litt.). Les 4 autres représentent pour l'ornithologue un ensemble bien différent : ce sont des Feuillus et/ou Conifères de basse et moyenne altitude et la structure simplifiée de la végétation les isole nettement du groupe franchement sylvatique des étages supraméditerranéen, montagnard et oroméditerranéen.

Les résultats que nous allons exposer ne sauraient préjuger de ce qui peut se passer ailleurs. Ils doivent donc être considérés comme strictement spécifiques à la situation du Ventoux. Tout au plus, certaines conclusions auxquelles nous aboutirons pourront-elles avoir une portée plus générale. Nous insistons sur ce point. 


\section{2. - MATÉRIEL ET MÉTHODES}

Nous ne nous étendrons pas sur le protocole d'échantillonnage qui a été développé ailleurs (BLondel, 1975). Précisons simplement que nous avons procédé à un échantillonnage stratifié de l'ensemble du versant sud et que les deux principaux critères retenus pour définir chaque strate d'échantillonnage sont la structure de la végétation et l'identité de la première ou des deux premières espèces végélales dominantes. Les documents utilisés pour définir, recenser et circonscrire les différentes strates ont été les cartes phytogéographiques de GoBerT et PAvTou (I969) et de BarBeno et al. (inédif) complétíes par des mesures et observations sur le terrain. Ces opérations destinées à définir des milieux de référence qui soient aussi homogènes que possible nous ont conduit a retenir les douze formations mentionnées en italiques sur le tableau 1. Au sein de chacune, un échantillonnage probabilisle a été effectué pour fixer les sfations où eurent lieu les relevés d'avifaune permettant d'établir la diagnose de chaque communauté.

Les précautions dont nous nous sommes entourés pour définir les milieux puis les stations nous autorisent à admettre que les résultats recueillis dans chaque formation sont représentatifs du peuplement d'oiseaux qu'elle abrite et nous n'aborderons pas iei les délicats problèmes d'homogénéité de l'échantillon qui dépasseraient le cadre de cette note.

Les relevés d'avifaune ont été effectués par la méthode des ḱchantillonnages fréquentiels progressifs (BLONDEL, 1975) qui permet, au terme de $N$ relevés élémentaires, de mesurer : $a$ ) le nombre $S$ d'espèces contactées au moins une foís ; b) la fréquence centésimale de chaque espèce $i$. définic comme le rapport du nombre de fois où l'espéce a été contactée $F A_{i}$ au nombre total de relevés : $F C_{i}=100 F A_{i} / N$,

Au total, I 82 relevés ont été répartis sur l'ensemble des douze milieux, soit une moyenne de 15,2 relevés par milieu.

Les paramètres utilisés dans cette étude sont :

$S$ : Richesse du milieu (nombre total d'espèces) :

$\bar{s}$ : Richesse moyenne, c'est-â-dire le nombre moyen d'espèces contactées î chaque releve;

$F C$ : Fréquence centésimale de chaque espéce ;

$H^{\prime}$ : Diversité informatique du peuplement qui est une mesure de la probabilité de contact de l'espèce $i$ dans la collection $T$ d'individus que se partagent les $S$ espèces. Les trois types de diversité informatique : alpha, béta et gamma que nous utiliserons seront définis plus loin:

$J^{\prime}$ : Equirépartition qui est le rapport de la diversité observée à la diversité maximale qui serait réalisée si toutes les espèces présentaient les mémes effectifs $\left(o<J^{\prime}<1\right.$ ).

Nous reportons le lecteur à notre précedent travail (BLosine, boe, cit.) pour plus amples renseignements sur la nature de ces paramétres et sur les relations d'ordre qui existent entro eux.

\section{3. - RÉSULTATS}

\section{I. - Richesse totale du territoire et nature de l'avifaune}

Les chiffres sont résumés sur le tableau 2. Les 12 formations sylvatiques représentent toutes ensemble un * univers forestier * contenant $5 \mathrm{I}$ espèces qui ont fourni I 760 contacts se répartissant de façon variable entre les espèces (de I contact à $I_{5} \mathrm{I}$ ). La diversité informatique de cet ensemble ou diversité gamma $\mathrm{H}_{\gamma}^{\prime}$ est 4,93 , chiffre dont la signification apparaîtra plus loin par comparaison avec d'autres diversités.

Un inventaire normalisé de l'avifaune nicheuse totale du Ventoux (cf. BLondis, I975) nous a permis de déterminer pour l'ensemble du massif 84 espèces nicheuses certaines. Avec $5 \mathrm{r}$ espèces, notre * univers forestier " compor ¿̇e donc plus de la moitié des espèces (6I p. Ioo) et une très grande majorité des individus (plus de 80 p. roo). En effet plusieurs espèces nicheuses sont très rares au Ventoux car elles habitent 
TABIEAU 2

Fréquences centésimales et amplitude d'habitat (cf. texte) des 31 espèces d'oiscaux dans les differentes formations forestieres du Mont Ventoux

\begin{tabular}{|c|c|c|c|c|c|c|c|c|c|c|c|c|c|}
\hline \multirow[b]{2}{*}{ Espéces } & \multicolumn{4}{|c|}{$\begin{array}{c}\text { Groupe I } \\
\text { Formations } \\
\text { spontanées des étages } \\
\text { montagnard et } \\
\text { oroméditerranéen }\end{array}$} & \multicolumn{3}{|c|}{$\begin{array}{l}\text { Groupe } 2 \\
\text { Reboisements } \\
\text { des étages } \\
\text { supramédit. } \\
\text { à oromédit. }\end{array}$} & \multicolumn{5}{|c|}{$\begin{array}{c}\text { Groupe } 3 \\
\text { Formations spontanées } \\
\text { et reboisements } \\
\text { des étages méditerranéen } \\
\text { à supraméditerranéen }\end{array}$} & \multirow{2}{*}{ 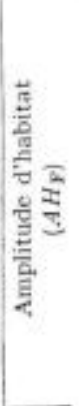 } \\
\hline & 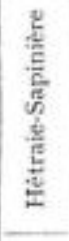 & 吾 & 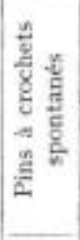 & 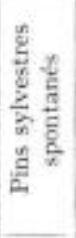 & $\frac{\mathrm{g}}{\mathrm{E}}$ & 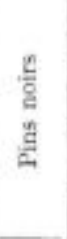 & 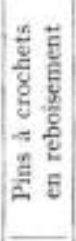 & 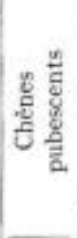 & 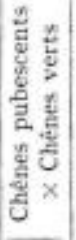 & $\begin{array}{l}\frac{0}{2} \\
\frac{\Phi}{4} \\
\text { है }\end{array}$ & $\begin{array}{l}\frac{0}{2} \\
0 \\
\frac{c}{2} \\
\frac{c}{2}\end{array}$ & 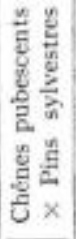 & \\
\hline $\begin{array}{l}\text { Epervier }, \ldots \ldots \ldots \ldots \ldots \\
\text { Autour }, \ldots \ldots \ldots \ldots \ldots \\
\end{array}$ & 6 & & & 10 & 6 & & & & & & & & 1,60 \\
\hline $\begin{array}{l}\text { Autour } \ldots \ldots \ldots \ldots \ldots \ldots \\
\text { Circaète } \ldots \ldots \ldots \ldots \ldots \ldots\end{array}$ & & & 6 & & 30 & 9 & & & & & & & $\begin{array}{l}1,66 \\
1,00\end{array}$ \\
\hline Pigeon ramier $. . . \ldots \ldots .$. & 12 & & & & 15 & 9 & & & & & 10 & 10 & 2,80 \\
\hline Tourterelle................ & & & & & 6 & & & 9 & 43 & 37 & 80 & & 2,32 \\
\hline Coucon $\ldots \ldots \ldots \ldots \ldots \ldots$ & 53 & 21 & & & 39 & 32 & 13 & & & 37 & 90 & & 5,07 \\
\hline Pic vert $\ldots \ldots \ldots \ldots \ldots \ldots$ & & & & & & & & & 6 & & & & 1, \\
\hline Pic épeiche ............. & 24 & & 50 & 40 & 30 & 50 & & & & & & & 2,77 \\
\hline Pic noir $\ldots \ldots \ldots \ldots \ldots \ldots$ & 29 & & & 20 & 3 & & & & & & & & 6 \\
\hline Alouette lulu...$\ldots \ldots \ldots$. & & & & & & & & & 43 & 50 & 50 & & 1,62 \\
\hline Corneille noire........... & & & & & & & & & & 12 & 10 & & 21 \\
\hline Pie $\ldots \ldots \ldots \ldots \ldots, \ldots$ & & & & & & & & & 6 & 50 & 10 & & 1,37 \\
\hline Geai $. . .+\ldots \ldots \ldots \ldots+\ldots+$ & $\$ 1$ & & 6 & & 54 & 27 & 13 & 45 & 56 & 50 & 60 & 30 & 7,52 \\
\hline Miésange charbonnière $\ldots$ & & 7 & 6 & 10 & 36 & 18 & & 18 & 81 & 87 & 20 & & 4,92 \\
\hline Mísange bleue. .......... & 6 & 28 & & & 9 & & & 36 & 25 & 25 & & 20 & 4,17 \\
\hline Mésange noire .......... & 9 & & 81 & 90 & 64 & 100 & 80 & 54 & & & 10 & 70 & 6,50 \\
\hline Mlésange buppće,$\ldots \ldots$. & 53 & & 87 & 50 & 54 & 59 & 67 & 18 & & & 90 & 10 & 6,52 \\
\hline Mésange nonnette ...... & 6 & 21 & & 10 & 3 & & & 9 & & & & 10 & 3,26 \\
\hline Mésange à longue queue .. & & & & & 24 & & & 27 & 25 & 37 & & & 2,15 \\
\hline Sittelle $\ldots \ldots \ldots \ldots \ldots \ldots$ & 18 & 16 & & & 9 & 41 & & & & & & & 1,98 \\
\hline Grimpereau des jardins ... & 82 & 21 & 56 & 60 & 82 & 95 & 13 & & 6 & & 60 & 50 & 7,11 \\
\hline Troglodyte............. & 76 & 21 & 87 & 100 & 70 & 82 & 67 & 36 & & & & 90 & 6,52 \\
\hline Grive draine...$\ldots \ldots \ldots$ & 6 & 7 & 19 & 30 & 21 & 18 & & & & & & & 3,30 \\
\hline Grive musicienne ........ & 6 & & & 3) & & & & & & & & & 1,13 \\
\hline Merle $\ldots \ldots \ldots \ldots \ldots \ldots \ldots$ & 65 & 57 & 56 & 39 & 100 & 41 & 27 & 82 & 87 & 100 & 100 & 60 & 11,36 \\
\hline Rouge-queue à front blanc. & 6 & & 12 & 10 & 6 & & & & & 25 & & & 2,57 \\
\hline Rossignol ,........... & & & & & 15 & & & 9 & 68 & 100 & & & 1,80 \\
\hline Ronge-gorge $\ldots \ldots \ldots \ldots$. & 100 & 93 & 100 & 100 & 97 & 73 & 93 & 91 & 37 & 11 & 100 & 80 & 11,13 \\
\hline Traquet oreillard ........ & & & & & & & & & 18 & 37 & & & 1,19 \\
\hline Fauvette à tête noire, .... & 92 & 14 & 44 & 80 & 91 & 41 & 60 & 73 & $6 \times$ & 62 & 10 & 160 & 10,59 \\
\hline Fauvette grisette ........ & & & & & & & & & & & & 20 & 1,00 \\
\hline Fauvette orphée & & & & & & & & 9 & 50 & 37 & & & 1,51 \\
\hline Fauvette mélanocéphale.. & & & & & & & & & 1. & 25 & 90 & & 1,65 \\
\hline Fauvette passerinette .... & & & & & & & & 27 & 8 & 100 & 10 & 10 & 2,27 \\
\hline Fauvette pitchou ........ & & & & & & & & & 18 & 50 & 200 & 10 & 1,99 \\
\hline Pouillot véloce,$\ldots . \ldots \ldots$. & 29 & 7 & 50 & 90 & 61 & 23 & 20 & 82 & 18 & & & 80 & 6,98 \\
\hline Pouillot de bonelli ....... & 12 & & 12 & & 25 & & & 65 & 87 & 50 & 40 & 10 & 4,69 \\
\hline Roitelet huppe .......... & 24 & & & 30 & 26 & & & & & & & & 1,61 \\
\hline Roitelet triple bandeau ... & 71 & & 5 & 70 & 65 & $5 i$ & 7 & & & & 10 & 90 & 6,85 \\
\hline Accenteur mouchet .... & 12 & & 94 & 30 & 36 & & 60 & 36 & 6 & & & 50 & 6,77 \\
\hline Pipit des arbres .... & & & & 10 & & & & & & & & & 1,00 \\
\hline Verdier $\ldots \ldots \ldots \ldots \ldots$. & & & & & 30 & & & & 18 & & & 10 & 1,56 \\
\hline Chardonneret ........... & & & & & 15 & & & & 6 & & 50 & & 1,61 \\
\hline Linotte $\ldots \ldots \ldots \ldots \ldots$ & & & & & 4) & 5 & & 9 & & & & & 1,58 \\
\hline Venturon...$\ldots \ldots \ldots$. & 12 & & 15 & & & & 27 & & & & & & 1,58 \\
\hline Serin cini $\ldots . . \ldots \ldots$ & & & & & 12 & & & & 37 & 37 & 90 & & 1,94 \\
\hline Bouvreuil .... & 29 & & & 60 & 3 & 4 & & & & & & & 1,74 \\
\hline Beccroisé....... & 12 & & 62 & 10 & 18 & 59 & 4 & & & & & 10 & 3,07 \\
\hline Pinson $\ldots \ldots \ldots \ldots \ldots \ldots$ & 100 & 65 & 100 & 100 & (100) & 100 & 93 & 100 & 25 & 50 & 100 & 100 & 11,67 \\
\hline Bruant ziyi $\ldots \ldots \ldots \ldots$ & & & & & & & & 9 & 18 & 87 & & & 1,76 \\
\hline Bruant fou,$\ldots \ldots \ldots \ldots$. & & & 31 & & & & & 18 & 12 & & 20 & 50 & 2,63 \\
\hline
\end{tabular}




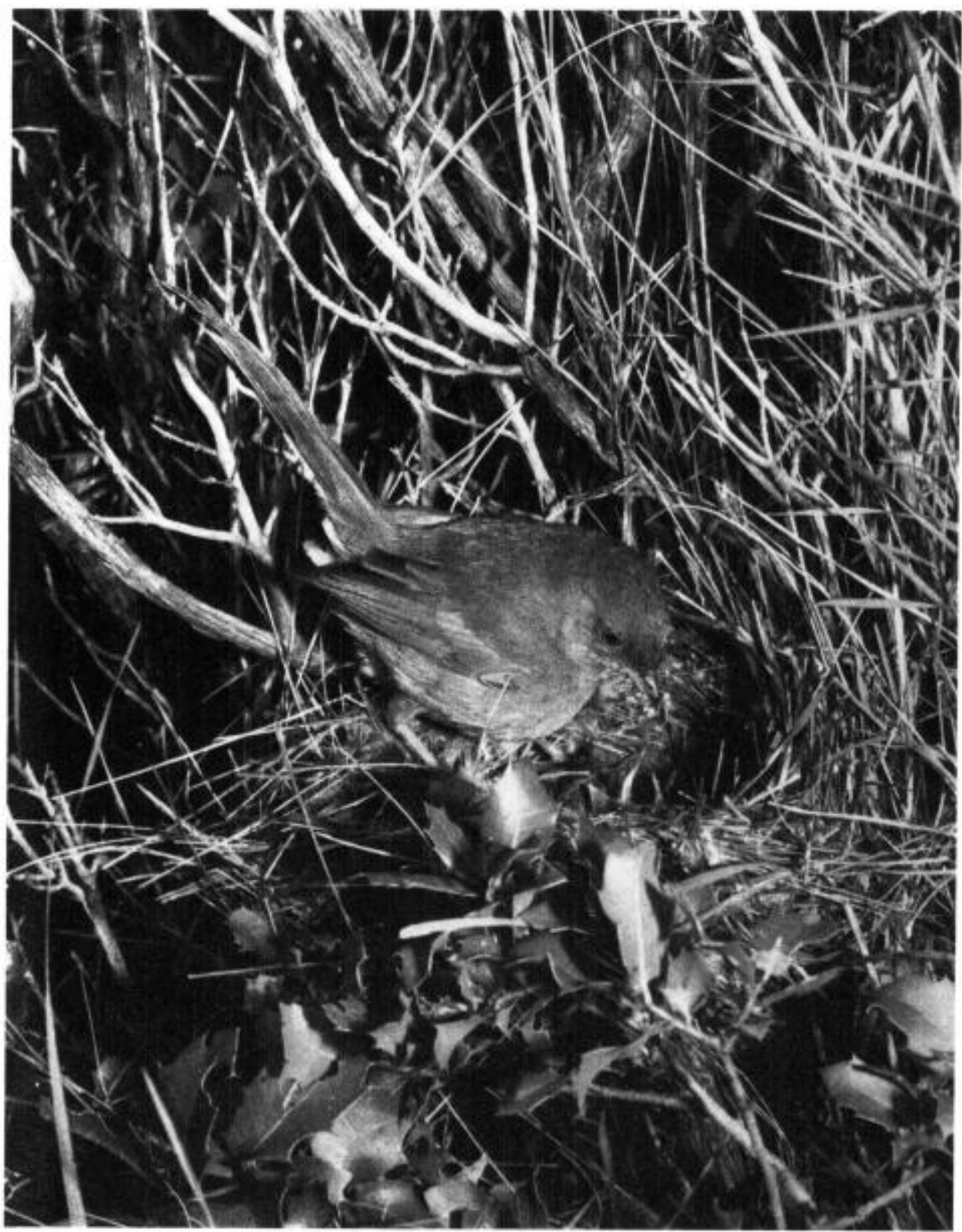

Fartetle pitchou : ojeau des milieux buissonnants bas : parrigurs ct landes 


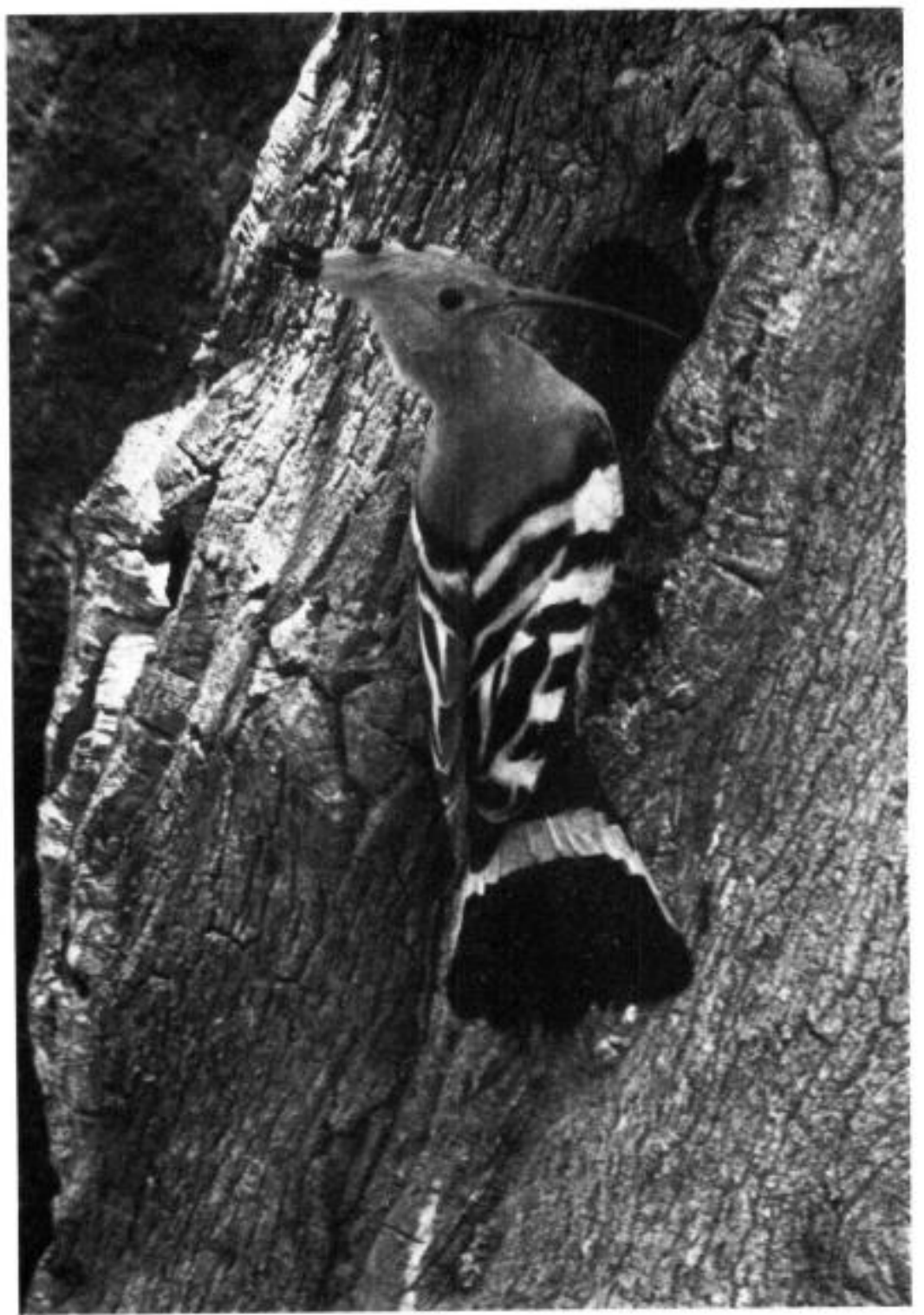

Huppe ; oicean des liseries, clatrieres et vieux vergers 


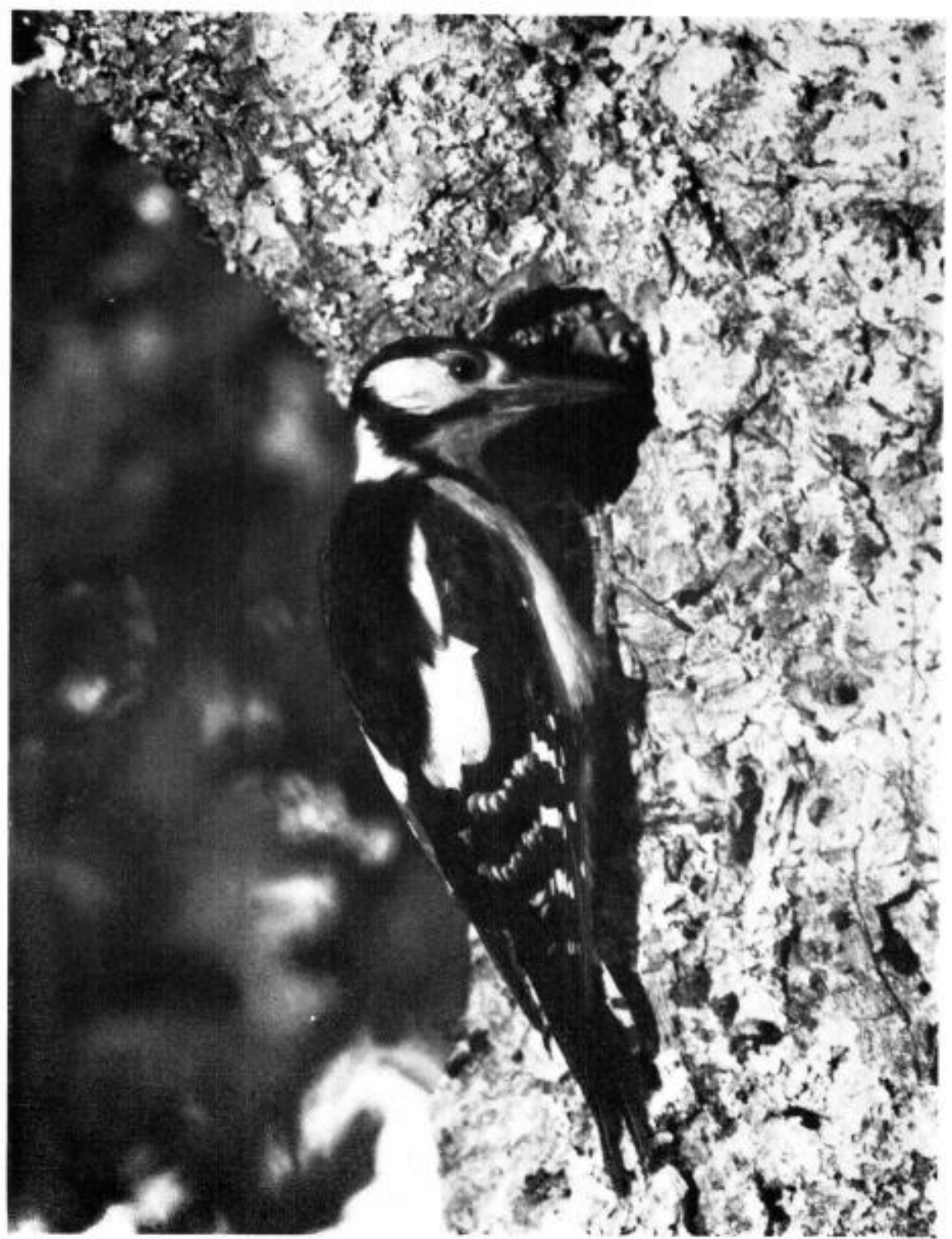

Pic priche : oisean typinuement forestier.

ce Pic est un bon indicateur du niveau de développement de la forèt 
des biotopes très pet représentés ou marginaux par rapport a l'ensemble du massif : biotope rupestre (Hibou grand-duc, Hirondelle de rochers), milieux cultivés et/ou habités du Piémont (Moineaux, Hirondelle de cheminée), ete. Ũn examen critique de la liste des espéces permet de faire la distinction entre les oiseaux qui habitent les milieux non franchement forestiers : milieux herbacés, milieux buissonnants, lisières, clairières, etc. de ceux qui sont spécifiquement sylvatiques. Les premiers

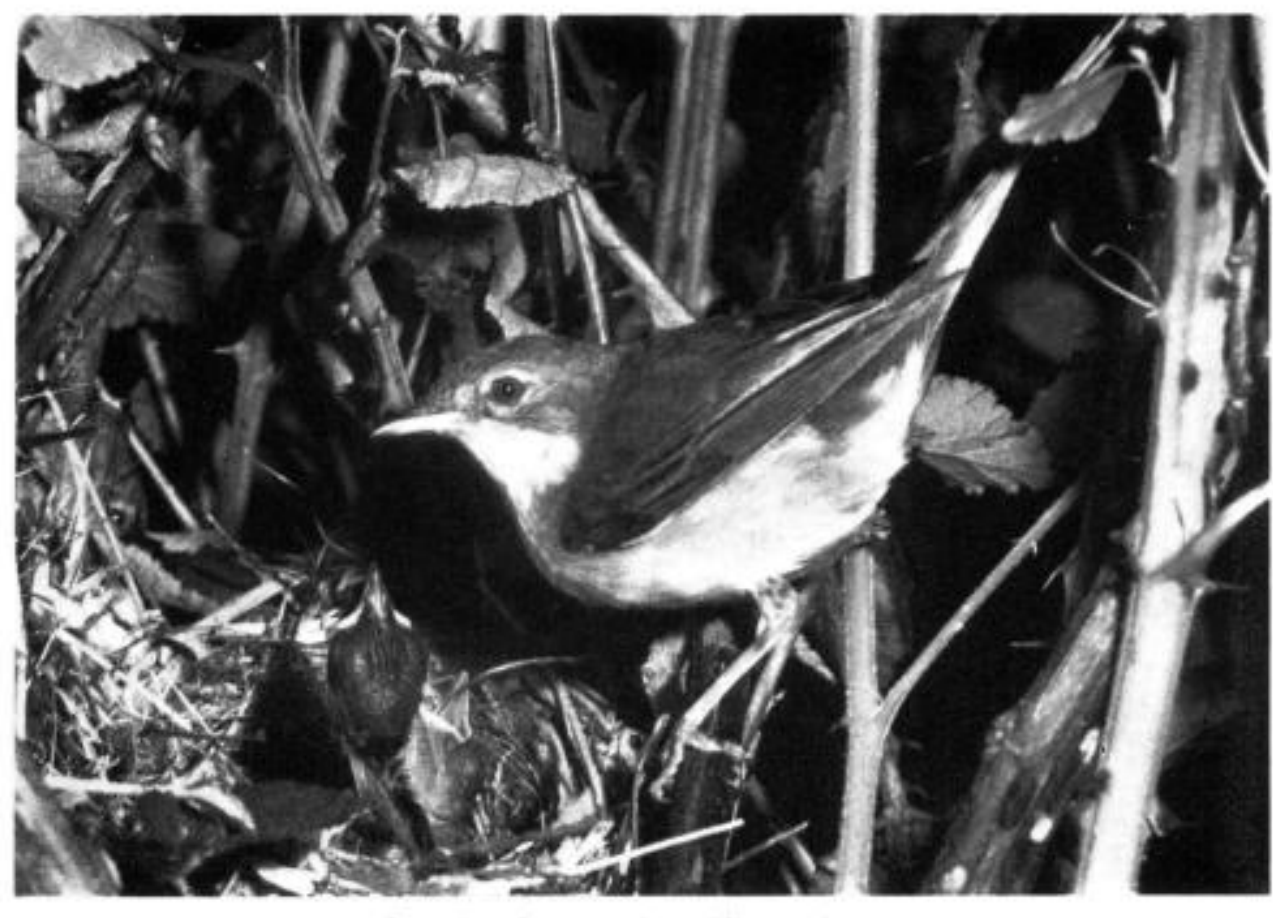

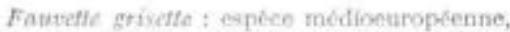

asses commune dans ies clairicres de Fitags supta-uácliterramien do Ventoux

sont au nombre de 13 ; ce sont certaines Fauvettes, les Traquets, les Bruants, ete. alors que tous les autres sont des oiseaux forestiers au sens strict c'est-à-dire pour qui l'arbre en tant que tel n'est pas un éément plus ou moins fortuit du paysage mais une composante nécessaire de l'habitat activement recherchée par l'oiseau parce qu'il s'y nourrit, y niche ou y chante (Mésanges, Pics, Roitelets). Ce premier constat permet déjà d'affirmer que le Ventonx a reconvré, un siècle après les premiers efforts de reboisement une avifaune proprement forestiere que nous allons maintenant étudier plus en détail.

Pour les besoins de l'argumentation, nous pouvons découper cet $\alpha$ univers forestier " en trois groupes et examiner la façon dont les espèces s'y distribuent :

- Groupe 1 : formations spontanées d'altitude moyenne à élevée, résineuses, mixtes ou caducifoliées des étages montagnard et oroméditerranéen : HêtraieSapinière, Hêtraie mésophile, Pineraie de Pins à crochets spontanée, Pineraie de Pins sylvestres. 
- Groupe 2: formations artificielles de résineux des étages supraméditerranéen à oroméditerranéen (Cédraie, Pineraie de Pins noirs, Pineraie artificielle de Pins à crochets).

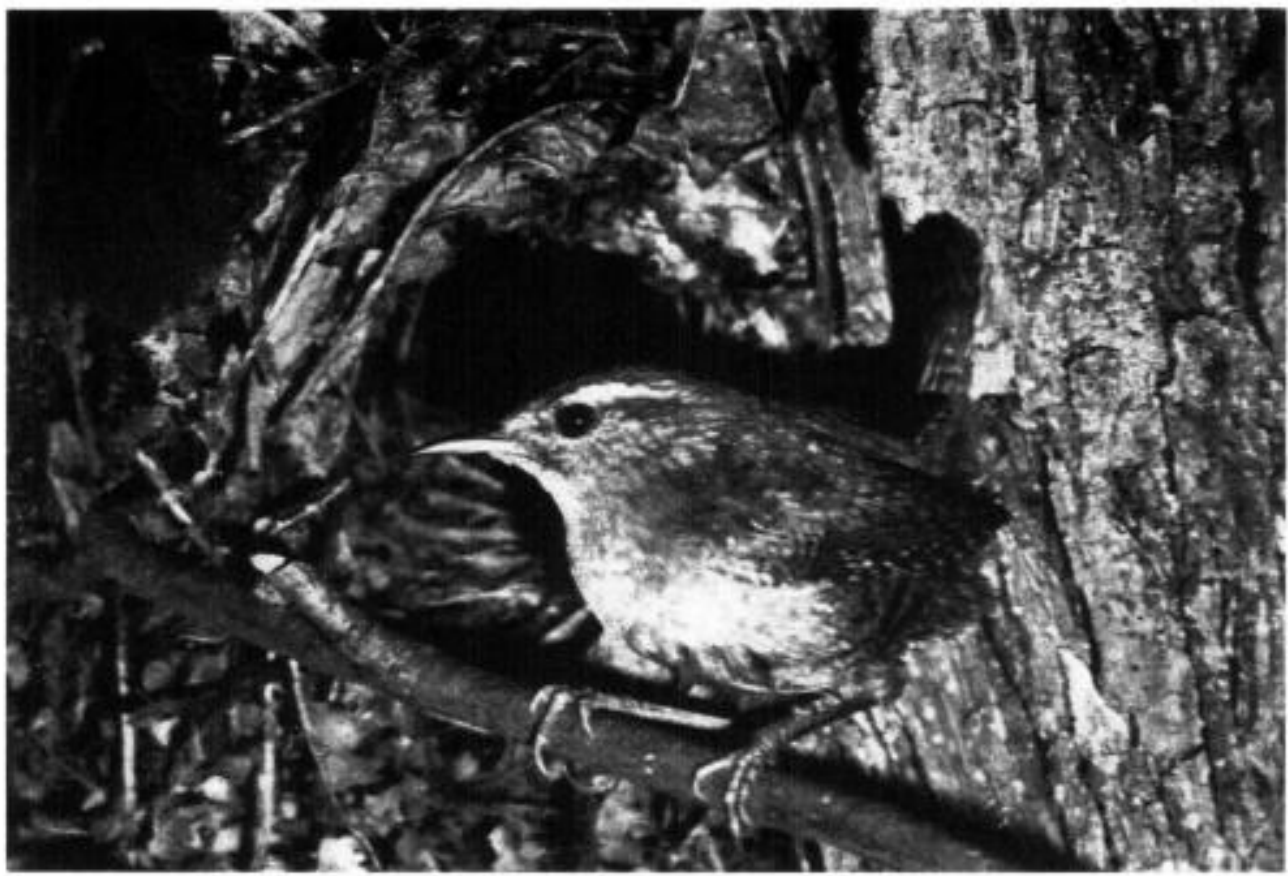

Trogladyle : espéce forestiêre habitant les strates basses de la végétation

- Groupe 3 : formations spontanées ou artificielles de feuillus et/ou de résineux des étages méditerranéen à supraméditerranéen : Chênaies de Chênes verts, de Chênes pubescents, Chênaies mixtes, Pineraies de Pins d'Alep, Bois mixtes de Chênes pubescents et de Pins sylvestres.

Une première approche d'ordre qualitatif consiste à comparer les peuplements des reboisements du $2^{\circ}$ groupe avec ceux des deux autres (tabl. 3 ).

TABLEAU 3

Repartition de l'avifaune forestize dis Ventoux par groupes de formations (cf. texte)

\begin{tabular}{c|c|c|c}
\hline \hline $\begin{array}{c}\text { Groupes de } \\
\text { formations }\end{array}$ & 1 & 2 & 3 \\
\hline 1 & 2 & 0 & \\
\hline 2 & 9 & 7 & 19 \\
\hline 3 & 1 & 20 & \\
\hline $1-3-3$ & & & \\
\hline
\end{tabular}


Sur les $5 \mathrm{I}$ espèces, deux setulement n'ont été rencontrées que dans les formations spontanées du premier groupe : ce sont la Grive musicienne, espèce médio-européenne en limite d'aire au Ventoux qui n'habite que les Pineraies de Pins sylvestres et la Hêtraie-Sapinière du versant nord et le Pipit des arbres, espèce de lisière et des clairières, voire des prés-bois, dont quelques couples ont été dénombrés dans les Pineraies claires de Pins sylvestres. Aucune espèce n'a été trouvée exclusivement dans les reboisements de Conifères mais 12 sont exclusives du $3^{e}$ groupe. Parmi celles-ci, II ont été trouvées dans les taillis de Chènes verts et de Chênes pubescents

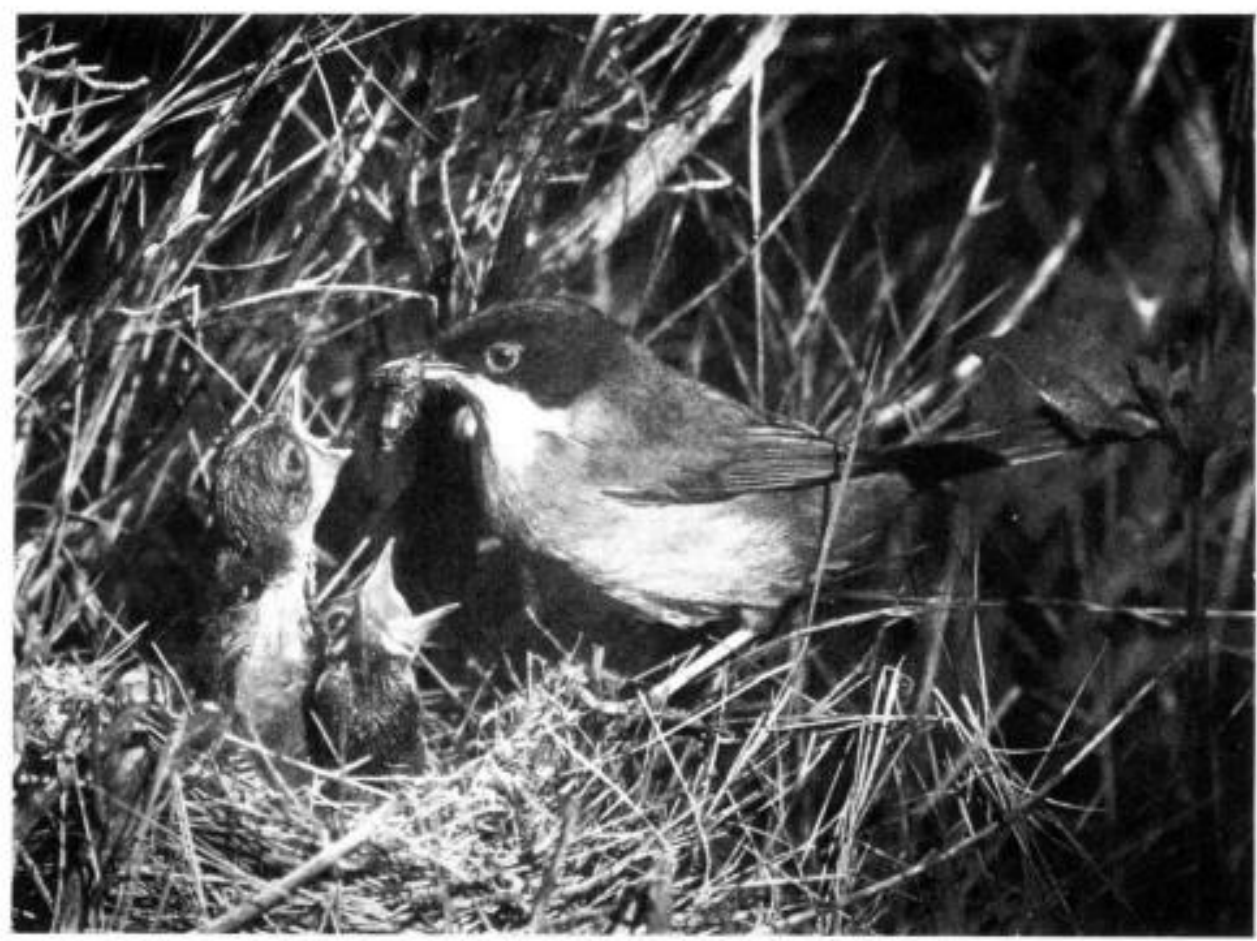

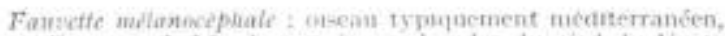
ciette Fauvette habite les sartizues chatedes dif pied du Ventoux

des étages méditerranéen et supraméditerranéen qui méritent à peine la dénomination de forêt. Ce sont en fait des oiseaux des milieux buissonnants dont le meilleur exemple est celui des Fauvettes méditerranéennes (Fauvettes orpháe, mélanocéphale, passerinette, pitchou). Cinq autres (Circaëte, Pic vert, Pie, Corneille, Bruant zizi) réagissent fortement à l'altitude et ne dépassent pas la cote $650 \mathrm{~m}$ en-deçà de laquelle ne se trouve aucun reboisement du $2^{\circ}$ groupe. Signalons au passage ce phénomène, que nous ne discuterons pas ici, d'une réduction progressive du nombre d'espèces par unité de surface avec l'altitude. La relation inverse est hautement significative avec $r=-0,91, \mathrm{P}<0,001$. La richesse spécifique décroit en moyenne de 0,8 espèce pour un gain de $200 \mathrm{~m}$ en dénivelée. Bref, l'effet de l'altitude et la structure de la formation s'allient pour individualiser nette- 


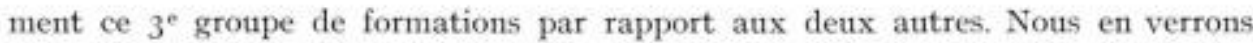
plus loin les conséquences à l'aide des coefficients d'affinité cénotique (analyse inter-habitats des peuplements).

Nous voyons par contre sur le tableau 3 que 9 espèces sont communes aux groupes I et 2,7 espèces aux groupes 2 et 3 et surtout 20 aux groupes 1,2 et 3 . Cela signifie qu'il existe un important brassage d'espèces puisque 36 , soit plus de $70 \mathrm{p}$. Ioo du total des espèces rencontrées dans l'une et/ou l'autre des formations spontanées se retrouvent dans les reboisements. D'où cette conclusion importante : globalement, les formations résineuses artificielles abritent à peu de chose près le même cortège d'espèces que les formations spontanées.

\section{2. - Les caractères collectifs propres à chaque peuplement analyse intra-habitats}

L'inventaire réalisé dans chaque milieu nous permet d'aller un peu plus loin et d'examiner les caractères collectifs des communautés (tabl, 4).

TABLEAU 4

Caractères collectifs des peuplements d'oiseanx des douse formations forestieres

\begin{tabular}{|c|c|c|c|c|c|c|c|c|c|c|c|c|}
\hline & HS & $\mathrm{Hm}$ & $\mathrm{PC}_{s}$ & PS & C & $\mathrm{PN}$ & $\mathrm{PCr}$ & $\mathrm{CP}$ & $\mathrm{CV} \cdot \mathrm{CP}$ & $\mathrm{CV}$ & PA & CP-PS \\
\hline$S \ldots$ & 28 & 13 & 21 & 23 & 155 & 21 & 15 & 29 & 28 & 93 & 23 & 29 \\
\hline $\bar{s} \ldots$ & 10,6 & 3,8 & 10,2 & 10,5 & 12,4 & 9,4 & 6,9 & 8,4 & 9,9 & 11,6 & 11,2 & 9,3 \\
\hline$H_{\alpha}^{\prime}$. & 4,29 & 3,23 & 4,03 & 4,15 & 4,63 & 6,01 & 3,58 & 5,06 & 5,38 & 4,33 & 4,12 & 4,05 \\
\hline$J^{\prime} \quad \ldots$ & 0,89 & 0,87 & 0,92 & 0,92 & 0,90 & 0,91 & 0,92 & 0,91 & 0,91 & 0,96 & 0,91 & 0,91 \\
\hline
\end{tabular}

Note : Signification des sigles comme sur le tableau 1 avec en outre : HS = Hêtraie-Sapinière : $\mathrm{Hm}=$ Ifétraie mésophile; $\mathrm{PCs}=$ Pineraie de. Pins à crochets spontanée; $\mathrm{PCr}=$ Pineraie de Pins à crochets en reboisement.

$S=$ Richesse totale $; s=$ Richesse noyenne ; $H_{\alpha}^{\prime}=$ Diversité informatique $; J^{\prime}=$ Bquirépartition.

\section{21. Le nombre d'espèces de chaque communauté.}

Le milieu le plus riche est la Cédraie avec 35 espèces alors que le plus pauvre est la Hêtraie mésophile du versant sud qui en contient deux fois moins. D'une façon générale, avec une moyenne de 23,7 espèces, les reboisements du groupe 2 ne sont pas moins riches que les formations naturelles du groupe $I$ qui en comptent en moyenne 21,2 ni que celles du groupe 3 qui en comptent 23,6 . On constate même que les reboisements paraissent plus riches que les formations spontanées. En fait, cela est dû aux performances exceptionnelles de la Cédraie que nous discuterons plus loin. Si nous raisonnons avec les richesses moyennes, nous avons un moyen de savoir si les différences constatées sont significatives. C'est ainsi que l'application du test $t$ de Student nous révèle que des trois formations résineuses artificielles, seule la Cédraie est significativement plus riche que chacune des 4 formations du groupe I $(\mathrm{P}<0,00 \mathrm{I})$. Par contre, les Pins à crochets en reboisement abritent les 
communautés les plus pauvres : leur richesse moyenne est statistiquement moindre que celle des formations spontanées sauf celle de la Hêtraie mésophile. Quant à la comparaison des nombres d'espèces des formations résineuses artificielles avec ceux des formations du groupe 3 , elle présente moins d'intérêt parce que les différences d'altitude dont nous avons évoqué plus haut l'importance oblitèrent en partie les résultats et parce que ces formations appartiennent à des étages de végétation fort différents.

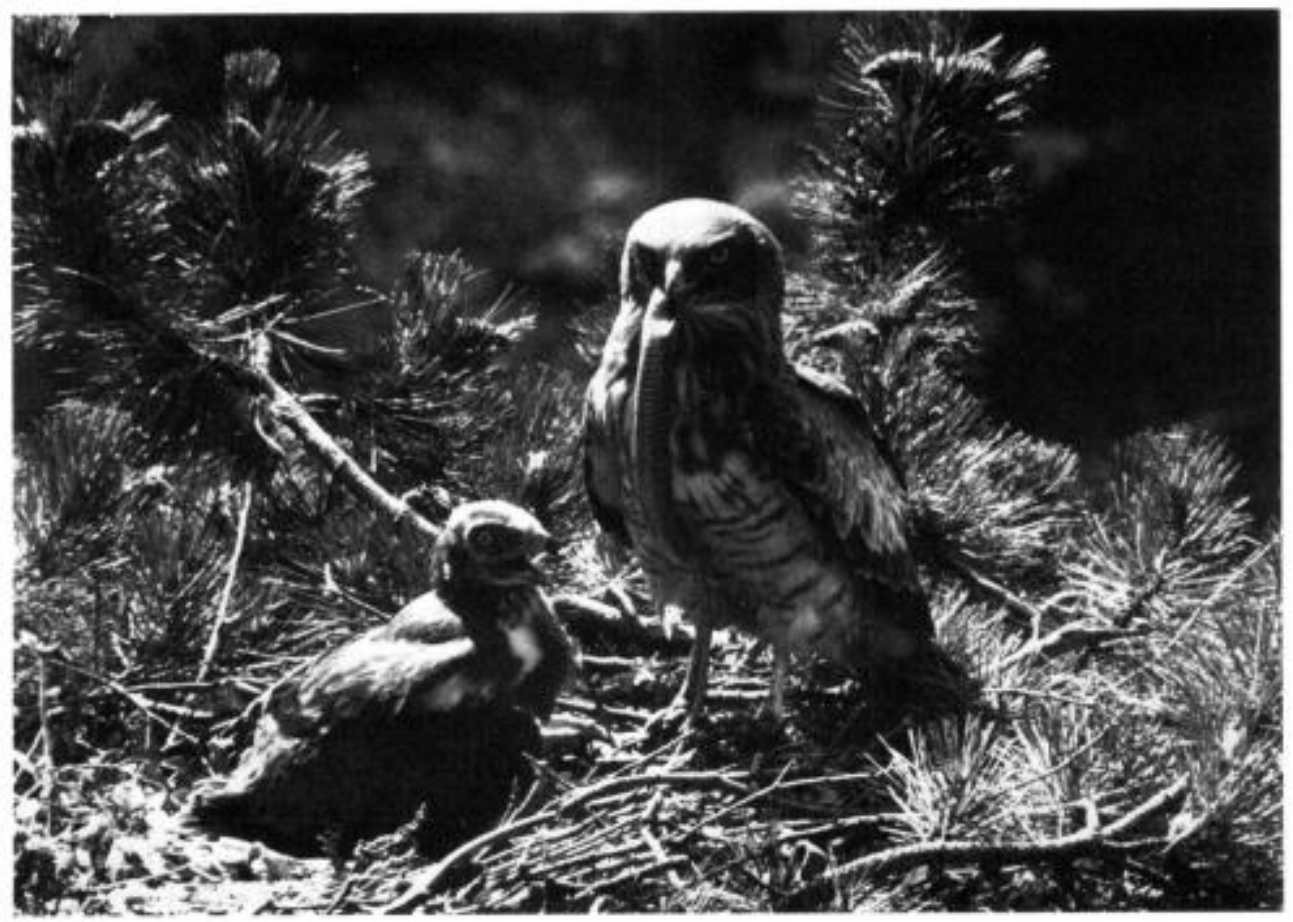

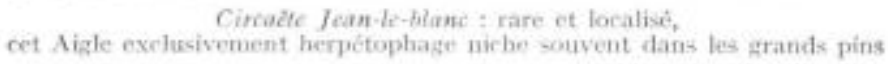

\section{22. La diversité dés communautés.}

Tout comme nous avions calculé la diversité d'ensemble de l'univers forestier $\left(H_{Y}^{\prime}=4,93\right)$, nous pouvons aussi définir dans chaque formation une diversité alpha $H^{\prime}$ ou diversité intra-habitat. Fille exprime la quantité d'information contenue dans chaque peuplement, en quelque sorte son niveau de complexité. Sans entrer dans les détails sur la nature et les propriétés de cet indice, disons simplement qu'à nombre d'espèces égal, plus l'indice est élevé, plus on a de chances que chaque oiseau observé appartienne à une espèce différente. Autrement dit, plus $H_{*}^{\prime}$ est élevé, plus la communauté est variée et plus le nombre de niches réalisées y est grand. Les valeurs de $H_{\alpha}^{\prime}$ consignées dans le tableau 4 montrent qu'aucun milieu n'est: plus diversifié que l'ensemble de l'univers puisque $H_{\alpha}^{\prime}$ est toujours inférieur à $H_{Y}^{\prime}$ : en moyenne, chaque formation apporte une contribution à la diversité d'ensemble du Ventoux, ce qui recoupe notre précédente conclusion sur le brassage des espèces dans les forêts du massif. Elles montrent aussi que les reboisements ne sont pas 
moins diversifiés que les formations spontanées : 4, 13 pour les premiers et 4,07 pour les secondes. On note même que, tout comme pour la Richesse, c'est la Cédraie qui détient le record, ce qui était d'ailleurs prévisible car on sait que la diversité et la richesse sont hautement corrélées (BLONDEL, I975). Cela signifie que la répartition d'ensemble des individus entre les différentes espèces est bien équilibrée. On peut mesurer ce degré d'équilibre en calculant l'indice d'équirépartition $J^{\prime}$ qui est une mesure du degré de réalisation de la diversité maximale potentielle. Les valeurs de $J^{\prime}$ sont très élevées puisqu'elles oscillent entre 0,87 et 0,96 . A titre de comparaison, l'indice d'équirépartition est bien plus bas dans les milieux physionomiquement simples comme les pelouses sommitales du Ventoux $\left(J^{\prime}=0,65\right)$ ou les milieux très dégradés comme certaines garrigues de Chênes Kermès $\left(J^{\prime}=0,52\right)$.

En résumé, les chiffres prouvent que les forêts du Ventoux, qu'elles soient spontanées on artificielles contiennent des communautés d'oiseaux diversifiées et équilibrées.

\section{23. La diversité des peuplements en fonction de la structure de la forêt.}

Dans un précédent travail (BLoNDEs, et al, I973) nous avons montré qu'il existe une relation linéaire entre la diversité des peuplements d'oiseaux et celle de la structure de la forêt, exprimée par un indice qui rend compte du degré de complexité de la répartition dans l'espace des éléments de végétation (masses de feuillage et branches). La méthode permettant de chiffrer l'indice de diversité de la végétation consiste à mesurer par l'analyse combinatoire les probabilités d'occurrence de la végétation dans des compartiments de l'espace limités par des * strates * dont les planchers sont conventionnellement fixés à des intervalles d'amplitude croissante de raison $2: 0,25 \mathrm{~m}, 0,50 \mathrm{~m}, 1 \mathrm{~m}$ etc.

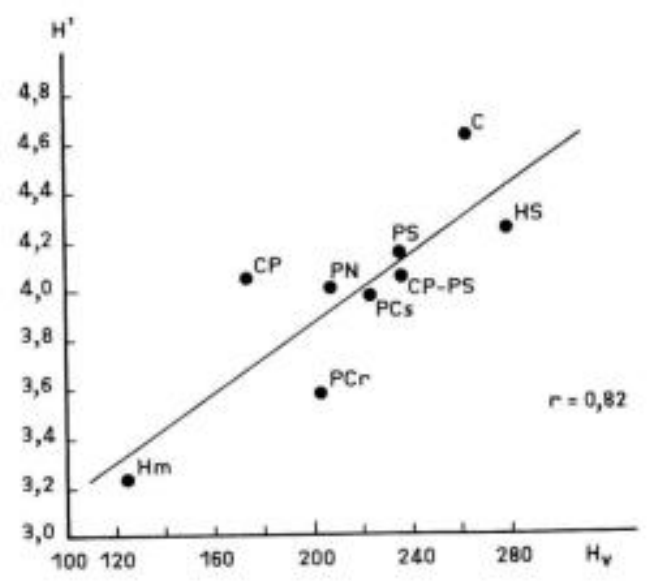

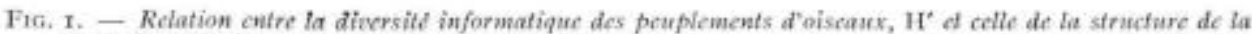
vegifation, $\mathrm{H}_{\mathrm{v}}$ (formations sylvatiques des étages supraméditerranéen, montagnard et oroméditerranéen). La signification des lettres est indiquile sur les tableaux 1 at 2.

Relation befween information theoretical dicersity $\mathrm{H}^{\prime}$ and diversity of structare of the wegetation fonly for the woodlands of supra-mediterranean to oro-mediterramean stages). The meaning of symbols is given in tables 1 and 2 .

Appliquée au cas du Ventoux, la méthode a permis de confirmer cette relation, 1a diversité des peuplements d'oiseaux étant directement proportionnelle à celle de la structure de la végétation : $r=0,82$, ( $P<0,001$, fig. 1 ). La position des reboi- 
sements par rapport à la droite de régression n'étant pas différente de celle des formations spontanées, on peut conclure que, considérés collectivement, les oiseaux réagissent de la même façon aux différentes formations forestières à structure simple de la végétation, avifaune peu diversifiée (Hêtraie mésophile, Pineraie artificielle de Pins à crochets) ; à structure complexe, avifaune riche et variée (HêtraieSapinière, Cédraie) peu importe que cette végétation soit spontanée ou artificielle.

\section{3. - Le partage de l'espace forestier entre les différentes espèces : analyse inter-habitats}

Chacune des $5 \mathrm{I}$ espèces recensées dans l'ensemble des forêts du Ventoux peut. être caractérisée par sa fréquence totale à l'échelle de l' * univers forestier * et par sa fréquence propre à chaque formation. L'analyse des distributions des fréquences spécifiques revient à faire une analyse inter-habitats des mécanismes d'occupation de l'espace forestier. Nous le ferons à deux niveaux : celui des espèces considérées individuellement et celui des communautés envisagées dans leur ensemble.

\section{31. Le partage de l'espace à l'échelle des espèces.}

Nous avons dressé les profils écologiques des 5 r espèces sur l'ensemble des formations et calculé l'amplitude d'habitat $A H_{P}\left(=c H_{x}^{\prime}\right)$ réalisée par chacune le long de ce profil. La façon dont on calcule l'amplitude d'habitat (les Anglo-Saxons disent * largeur de niche i) est exposée ailleurs (BLONDEL, r975) et nous ne reviendrons pas sur les fondements mathématiques de cet indice qui dérive, lui aussi, de la théorie de l'information. Rappelons simplement ce qui nous intéresse ici, à savoir que l'indice varie entre $l$ lorsque l'effectif total de l'espèce se trouve dans une seule classe d'habitat (une seule formation) et le nombre total de classes, soit I2 dans le cas présent, si l'espèce présente les mêmes effectifs dans chacune (équirépartition parfaite). Autrement dit, un oiseau dont $A H_{F}=1$ est un spécialiste parfait puisqu'il ne se trouve que dans un seul milieu alors qu'un oiseau dont $A H_{F}=\mathrm{I} 2$ serait un généraliste parfait, indifférent à la nature de la forêt.

Les amplitudes d'habitat sont indiquées sur le tableau 2 au regard de chaque espèce. Flles conduisent à d'intéressantes conclusions :

- Quatre espèces seulement ont une amplitude d'habitat de I; elles n'ont donc été rencontrées que dans un seul milieu ; ce sont la Fauvette grisette, le Circaëte, le Pic vert et le Pipit des arbres. Or, toutes présentent des fréquences très faibles (entre 6 et 27), soit qu'elles aient été sous-échantillonnées du fait de leur rareté (Circaëte), soit plutôt qu'elles ne soient pas vraiment forestières (Fauvette grisette, Pipit des arbres) soit enfin qu'elles réagissent fortement aux altitudes inférieures (Pic vert). Bref, toutes ont de bonnes raisons d'être marginales dans le cortège d'oiseaux du Ventoux.

- A l'opposé, 4 espèces (Merle, Rouge-gorge, Fauvette à tête noire, Pinson) ont une amplitude supérieure à Io ; à peu de chose près, elles peuvent être considérées comme ubiquistes car on les trouve dans tous les milieux forestiers français dont elles constituent le fond de l'avifaune, qu'il s'agisse de forêts de plaine ou de montagne, de feuillus ou de résineux.

- Toutes les autres présentent une amplitude d'habitat moyenne. Leur examen ne permet pas a priori d'établir une classification particulière. 
Par contre, si l'on calcule par formation la moyenne des amplitudes d'habitat des espèces qui la peuplent, on a le moyen de préciser un degré de spécialisation de l'avifaune (tabl. 5).

\section{TABIFAU 5}

Moyenue des amplitudes d'habitat des especes dans les 12 formations forestières du Venfoux

\begin{tabular}{|c|c|}
\hline Formation & $\begin{array}{l}\text { Amplitude } \\
\text { moyenne } \\
\text { d'habitat }\end{array}$ \\
\hline Hetraie-Sapinière *,. & 4,48 \\
\hline Hétrate mesophile $\ldots \ldots \ldots \ldots \ldots \ldots \ldots \ldots \ldots \ldots \ldots \ldots$ & 6,65 \\
\hline Pineraie spontance de Pins à crochets............. & 6,03 \\
\hline Pineraie de Pins sylvestres.$\ldots \ldots \ldots \ldots \ldots \ldots \ldots$. & 5,05 \\
\hline 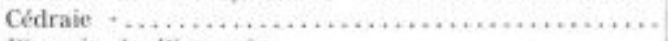 & 4,38 \\
\hline 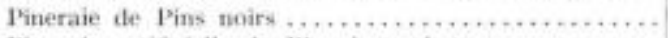 & 5,59 \\
\hline Pineraie artificielle de Pias à erochets............. & 7,25 \\
\hline Chernaie de Chernes pubescents $+\ldots \ldots \ldots \ldots \ldots \ldots$ & $5, \pm 8$ \\
\hline Chênaje thixte de Ch, verts et $\mathrm{Ch}$, pubescents ....... & 6.10 \\
\hline Chènaie de Chénes verts , . . . . . . . . . . . . . . . . . & 6.10 \\
\hline 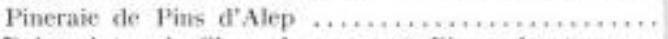 & 4,88 \\
\hline Rois mixtes de Ch. pubescents et Pins sylvestres.... & 5,58 \\
\hline
\end{tabular}

Il ressort de ce tableau que parmi les formations sylvatiques d'altitude, c'est la Cédraie qui possède le cortège le plus spécialisé ou plutôt qui comporte le plus d'espèces exigentes dans le choix de leur habitat comme le Pic noir, l'Autour, la Sittelle, le Roitelet huppé et le Bouvreuil par exemple qui ont tous une faible amplitude d'habitat. A l'inverse, c'est la Pineraie de Pins à crochets en reboisement qui possède le cortège le moins spécialisé. Tout se passe comme si ce dernier milieu dont la structure est simple et peu diversifiée n'était en mesure d'accueillir que les espèces les moins difficiles dans la sélection de leur habitat, c'est-à-dire les ubiquistes, notamment les 4 espèces énumérées plus haut.

Dans le cas général, nous avons trouvé une relation inverse entre le nombre d'espèces qui peuplent une formation et l'amplitude moyenne de leur habitat $(r=$ $-0,82, \mathrm{P}<0,00 \mathrm{I}$, fig. $2 a$ ). Autrement dit, les premières espèces à coloniser le milieu sont les plus banales, celles que l'on trouve partout, puis au fur et à mesure que le peuplement devient plus complexe, il s'y ajoute de nouvelles espèces plus spécialisées ou plus rares. La relation démontrée plus haut entre la diversité du peuplement d'oiseaux et celle de la structure de la forêt permet de dire que plus le paysage forestier est diversifié, plus il est apte à accueillir les espèces rigoureuses dans le choix de leur habitat. La démonstration peut en être apportée par le calcul du coefficient de corrélation entre l'amplitude moyenne d'habitat et la diversité de structure du paysage végétal ; nous avons trouvé $r=-0,64(\mathrm{P}<0,05$, fig. 2 b).

L'exemple des formations de Pins à crochets est très démonstratif de ce phénomène parce qu'elles sont représentées au Ventoux sous leur forme spontanée et en reboisements. L'amplitude moyenne d'habitat des espèces des Pineraies spontanées est de 6,03 alors qu'il atteint 7,25 dans les reboisements (différence significative au 
seuil de 5 p. roo). Sont en effet éliminées de ces derniers les espèces qui ont besoin pour s'installer de certaines caractéristiques de la forèt absentes des reboisements : troncs, espaces dégagés, sous-bois, etc. qui permettent dans les Pineraies spontanées l'implantation d'espèces assez exigentes comme l'Atutour, le Pic épeiche, le Rouge queue à front blanc, le Pouillot de Bonelli et le Bruant fou.

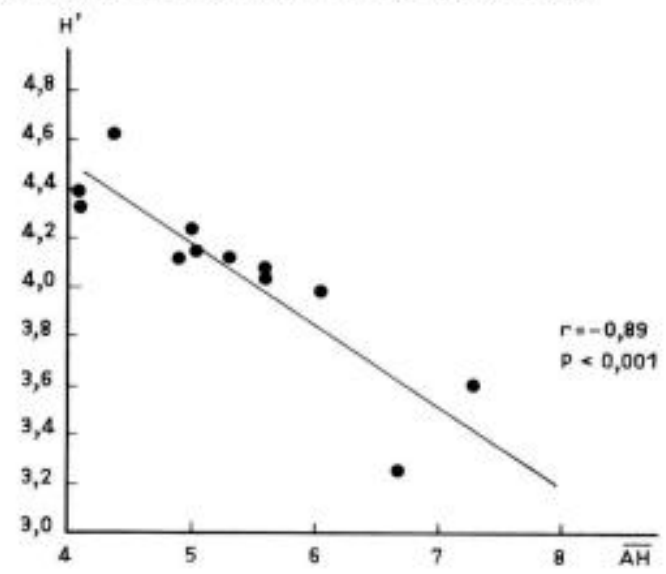

a

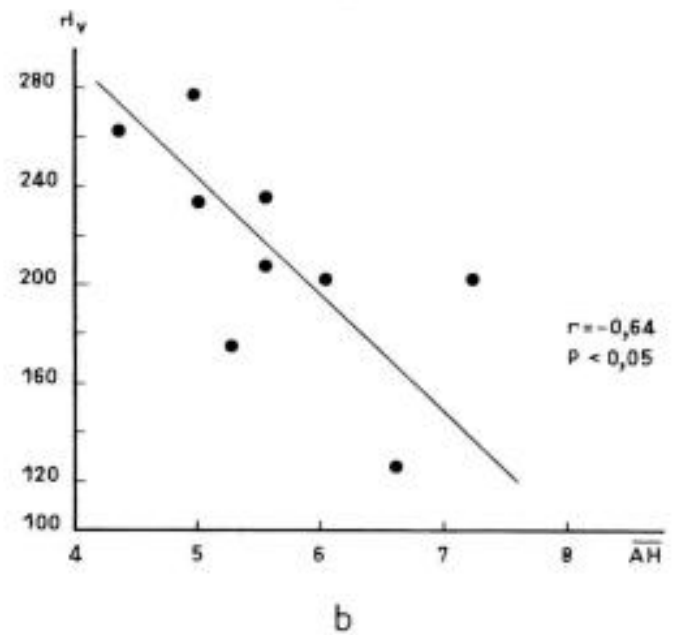

Fic. 2. - a : relation entre la ditersite informatique $\mathrm{H}^{\prime}$ des peuplements d'oiscaux d Famplitude d'habitat moyenne des espices $\mathrm{AH} ; \mathrm{b}$ : velation entre la structare de la végetation $\mathrm{Hv}$ a lasplatude d'habitat moyesne $\mathrm{AH}$ (étages supraméditerranécn, montagnard et oro-méditerranéen) ; ff. texte.

retalion between information-theordical disursity $\mathrm{H}^{\prime}$ of bird communities and wean nicke breadth (Alf) of the species: $\mathrm{b}$ : relation befween diversity of structure of the wegetation and mian niche breadlh of the species (supra-mediterranean to oro-mediterranean stages); cf. fext.

3. 32. Le partage de l'espace à l'échelle des communautés.

Les données quantitatives que nous avons acquises nous permettent de chiffrer le niveau de ressemblance entre deux communautés. Des différents coefficients d'affinité disponibles, nous avons choisi le plus performant d'entre eux : la diversité 
bêta, $\mathrm{H}_{\beta}^{\prime}$ qui est notre troisième type de diversité ou diversité intcr-habital. Cêtte diversité exprime la quantité d'information apportée par la différence de structure (et non pas seulement de composition) entre deux peuplements. Elle varie entre 1 (les 2 communautés sont totalement différentes et ne possèdent aucune espèce en commun) et o (toutes les espèces sont communes aux deux peuplements et y présentent les mêmes effectifs). Cet indice se calcule par la formule : $H_{\mathrm{B}}^{\prime}=H_{\alpha_{12}}-0,5$ $\left(H_{\alpha_{1}}^{\prime}+H_{\alpha_{2}}^{\prime}\right)$. Le niveau de ressemblance est donc $\mathrm{I}-H_{\beta}^{\prime}$. Ies valeurs de ces coefficients propres aux I2 formations prises 2 à 2 figurent sur le tableau 6.

TABIEAU 6

Cocfficients d'affinite $\left(\mathrm{r}-\mathrm{H}_{\beta}^{\prime}\right)$ des 12 formations sylyatiques du Mont Ventoux, prises deux à deux

\begin{tabular}{|c|c|c|c|c|c|c|c|c|c|c|c|}
\hline & HS & $\mathrm{Im}$ & $\mathrm{PCs}$ & PS & c & PN & $\mathrm{PC}$. & $\mathrm{CP}$ & $\mathrm{CV}-\mathrm{CP}$ & $\mathrm{CV}$ & PA \\
\hline HS ....... & - & & & & & & & & & & \\
\hline $\mathrm{Hm}, \ldots .$. & 0,51 & - & & & & & & & & & \\
\hline $\mathrm{PC} s, \ldots \ldots$ & 0,82 & 0,98 & - & & & & & & & & \\
\hline PS $\ldots . . .$. & 0,88 & 0,67 & 0,85 & - & & & & & & & \\
\hline$c, \ldots \ldots \ldots$ & 0,87 & 0,60 & 0,80 & 0,79 & - & & & & & & \\
\hline PN ,...... & 0,89 & 0,56 & $0,8.5$ & 0,80 & 0,82 & - & & & & & \\
\hline $\mathrm{PCr}, \ldots \ldots$ & 0,75 & 0,60 & 0,85 & 0,73 & 0,68 & 0,78 & - & & & & \\
\hline $\mathrm{CP} \ldots \ldots$ & 0,68 & 0,56 & 0,72 & 0,66 & 0,74 & 0,62 & 0,70 & - & & & \\
\hline $\mathrm{CV} \cdot \mathrm{CP} \ldots$ & 0,39 & 0,28 & 0,37 & 0,32 & 0,56 & 0,13 & 0,28 & 0,67 & - & & \\
\hline $\mathrm{CV}, \ldots \ldots$ & 0,35 & 0,25 & 0,26 & $0, \pm 3$ & 0,67 & 0,27 & 0,22 & 0,57 & 0,89 & - & \\
\hline $\mathrm{PA} \ldots \ldots$ & 0,57 & 0,43 & 0,58 & 0,62 & 0,62 & 0,55 & 0,57 & 0,56 & 0,65 & 0,62 & - \\
\hline CP-PS..... & 0,80 & 0,54 & 0,85 & 0,78 & 0,78 & 0,75 & 0.81 & 0,85 & 0,50 & 0,35 & 0,55 \\
\hline
\end{tabular}

La valeur moyenne des 66 coefficients obtenus est de o,60 ce qui est assez élevé et confirme nos conclusions précédentes sur le brassage des avifaunes de l'univers forestier du Ventoux. Plus intéressant est de chercher à savoir si les reboisements en conifères se ressemblent davantage entre eux qu'avec les formations spontanées. La moyenne des coefficients de similitude des trois reboisements est de 0,75 et celle de chacun des reboisements avec les 4 peuplements du groupe I (HS, Hm, PS, PCs) est respectivement de 0,72 pour la Cédraie, 0,78 pour la Pineraie de Pins noirs et 0,73 pour les Pins à crochets en reboisement. Ces valeurs étant très voisines, on en conclut que les reboisements n'ont pas " sélectionné a un type d'avifaune qui les individualiserait par rapport aux autres forêts. 
Les relations * inter-peuplements " qu'expriment ces coefficients d'affinité peuvent être visualisées de façon synthétique et démonstrative par la construction d'un dendrogramme de communanté (fig. 3. cf. par exemple DAGET, I975 pour la façon dont on construit un dendrogramme). Ainsi apparait une hiérarchie qui rend compte en quelque sorte des s parentés in entre ces différentes forêts tout comme le ferait un arbre généalogique. Ce dendrogramme conduit à plusieurs conmentaires ; nous nous limiterons à ceux-ci :

- ainsi qu'on pouvait s'en douter au vu de ce qui précède, les trois reboisements ne forment pas un lot à part :

- ainsi qu'il était suggéré par nos précédentes discussions, le dendrogramme sépare nettement les trois formations de l'étage méditerranéen des formations supraméditerranéennes et montagnardes et isole la Hêtraie mésophile, probablement en raison de la grande pauvreté de ce milieu ;

- huit milieux reliés au niveau de similitude 0,75 forment un ensemble homogène plus particulièrement les six foréts résineures de montagne artificielles et spontanées dont les coefficients de similitude sont compris entre 0,77 pour la branche qui les supporte et 0,89 entre la Hêtraie-Sapinière et la Pineraie de Pins noirs.

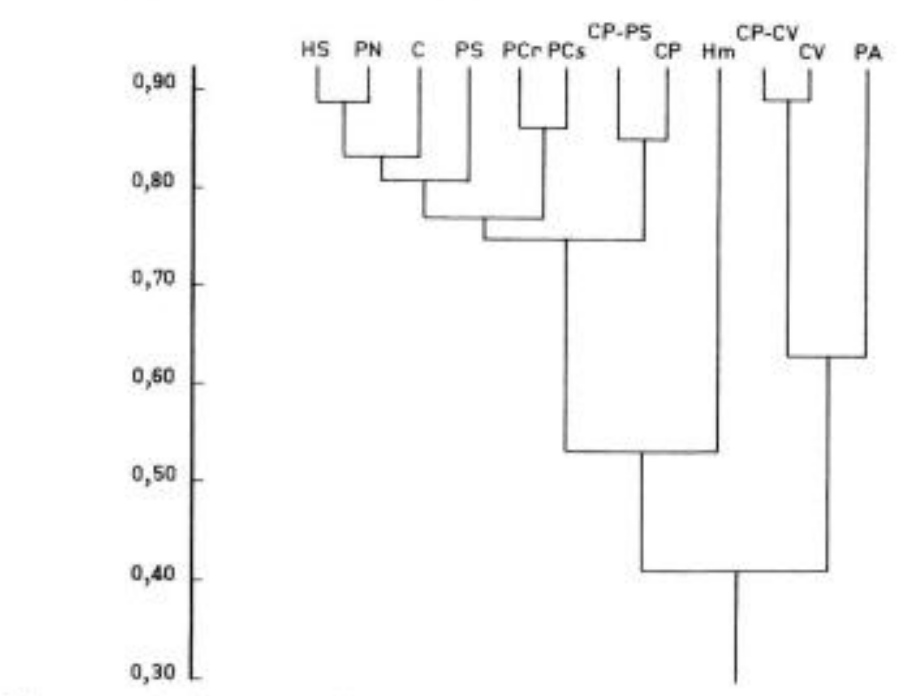

Fic. 3. - Dendrogramene de communastés crprimant les nizcatux de ressembiance des 12 peuplanents (cf, texte)

Commanity dendrogram shereme the ecological relationships of the 72 bird communities $[\mathrm{cf}$. text)

\section{4. - DISCUSSION ET CONCIUSION}

Les résultats dont nous venons de faire état nous amènent à reconnaitre objectivement que les travaux de reboisement ont reconstruit au Ventoux des communautés d'oiseaux probablement assez voisines de ce qu'elles devaient etre avant les mutilations ciont elles ont été victimes. Il est malheureusement très difficile de savoir 
quelle serait la nature authentiquement spontanée de ces peuplements car la plupart des forêts françaises ont été exploitées depuis si longtemps que le stade climacique a presque partout disparu, nous privant de ce fait d'éléments de comparaison.

Pour vivre et se reproduire dans un milieu, l'oiseau a une série d'actes nécessaires à accomplir. Les plus évidents sont : $I^{\circ}$ la recherche de la nourriture dans un environnement végétal qui soit adapté à ses techniques de chasse et qui contienne une nourriture suffisante $; 2^{\circ}$ 1'activité vocale qui est nécessaire à la défense du territoire et à la cohésion du groupe familial ; cette activité exige un poste de chant convenable ; $3^{\circ}$ l'acte de la reproduction proprement dit qui nécessite des matériaux de construction du nid et un site de nidification approprié. Ces activités se déroulent dans le décor forestier et au moyen de lui mais selon des modalités différentes suivant chaque espèce. On dit que chacune occupe l'espace écologique à sa propre manière. Plus cet espace écologique est varié, plus il y aura de manières possibles de l'occuper, plus il y aura de niches écologiques, donc d'espèces différentes. Nous avons démontré ce phénomène par la relation entre la diversité de structure de l'espace forestier $\left(H_{v}\right)$ et la diversité des peuplements d'oiseaux $\left(H^{\prime}\right)$.

Prenons l'exemple de la forêt où les deux diversités, celle de la végétation et celle des peuplements d'oiseaux sont les plus élevées : la Cédraie. Un siècle après le noyau initial d'une quarantaine d'ha semé par l'inspecteur Trchadou, la forêt couvre aujourd'hui plus de 300 ha. C'est une $\alpha$ futaie jardinée mélangée par larges bouquets entrecoupés de taches très denses et très vigoureuses de semis, fourrés, gaulis et perchis ^ (ARRIGHI, I972) dans laquelle on trouve quatre générations d'arbres. « Le massif est une véritable forêt subspontanée dont les strates inférieures arbustive, herbacée et muscinale restent celles des massifs voisins de Chênes pubescents a (GoBerT et PAUTou, I969) avec une représentation importante de Buis, Érable opale, Amélanchier, Cytise, Coronille, etc.

Toutes les conditions d'une ambiance forestière favorable aux oiseaux sont réunies : présence de feuillus et de conifères, coexistence de buissons et de très grands arbres, port de ces derniers dont les grosses branches présentent un angle d'attache ouvert avec le tronc et qui sont séparées par de vastes espaces libres où les oiseaux peuvent circuler, écorce crevassée où les insectes trouvent refuge, structure du feuillage favorable à la nidification de plusieurs espèce, etc.

Bref, la diversité floristique et la diversité de structure de la forêt créent dans la Cédraie la gamme complète des habitats de notre avifaune forestière qui comporte des grands Rapaces arboricoles (Autour), des oiseaux qui creusent leur loge dans les arbres (Pic noir et épeiche), des espèces de buissons (Fauvettes, Pouillots), des espèces qui se nourrissent et nichent dans les branches mortes dont le rôle est important (Rouge queue à front blanc, Mésange huppée) et tout le cortège des oiseaux qui nichent et se nourrissent dans les arbres (Mésanges, Sittelles, Grimpereaux, Fringilles) en se partageant l'espace suivant des normes précises qu'il serait trop long de discuter ici. Soulignons que la coexistence de feuillus et de conifères augmente la diversité d'ensemble parce qu'aux espèces liées au feuillus qui ne sont pas éliminées (Mésange bleue, Pouillots) s'ajoutent des espèces caractéristiques des conifères qu'attirent le Cèdre ainsi que les Pins noirs et Pins sylvestres qui s'y trouvent en mélange. C'est le cas des Mésanges huppée et noire et surtout du Roitelet huppé, du Venturon et du Bec-croisé absents des Chênaies de Chênes pubescents voisines,

Tout cela explique pourquoi la Cédraie du Ventoux est le milieu forestier le 
plus riche que nous ayons en l'occasion d'étudier en France méditerranéenne et probablement parmi les plus riches de France. L'ornithologue ne peut que joindre sa voix à tous ceux qui ont vigoureusement plaidé la cause du Cèdre en région méditerranéenne (cf. ToTH, 1972 ).

A l'opposé, les monocultures équiennes de conifères sont beaucoup plus pauvres en raison de la faible diversité de structure de la végétation et de l'appauvrissement du cortège floristique qui réduisent considérablement le nombre de niches écologiques. Ces tristes boisements monotones et sombres où les arbres plantés trop serrés ne laissent pas pénétrer la lumière sous les houppiers bas et jointifs éliminent en priorité les espèces plus délicates dans le choix de leur habitat comme nous l'avons montré par la relation inverse entre l'amplitude d'habitat des espèces et la diversité de la structure de la forêt. Plus la physionomie du décor forestier devient simple et homogène, plus l'avifaune se banalise car il ne subsiste plus que quelques espèces très ubiquistes que l'on rencontre partout car elles sont capables de s'accommoder d'une forte altération de leur habitat. La démonstration en est apportée ici par les peuplements des reboisements en Pins noirs bien plus pauvres que ceux de la Cédraie et surtout par ceux des Pineraies de Pins à crochets que nous avons eu la chance de pouvoir comparer avec ceux des formations spontanées de la mème essence ( $\mathrm{f}$. supra). Si de tels boisements sont parfois nécessaires pour reconstituer un sol et préparer le retour d'une véritable forêt, ils ne devraient toujours être qu'un stade transitoire dans l'histoire forestière d'une région.

Reģu pour publication en juillet I9ז6.

\title{
REMIRCIIMMENS
}

L'auteur tient à remercier tout particuliêrement MM. C. FErry, B. Frochot et J. PArdE qui ont bien voulu relire le manuscrit et y apporter d'intéressantes suggestions ainsi que P. ISENMaxs qui a traduit le résumé allemand.

\author{
SUMMARY
}

\author{
THE ECOLOGICAI, INCIDINCE OF REAFFORISTATION \\ ON BREEDING BIRD COMMUNITIES : \\ THE CASE OF MONT VENTOUX (SOUTH OF FRANCE)
}

An ecological diagnosis of breeding bird communities has been carried out in twelve native and artificial forests in the Mont Ventoux (south of France). The results show that :

- $5 \mathrm{I}$ species, namely $6 \mathrm{I}$ p. 100 of the brecding avifauna of the mountain actually brecd in woodland landscape. The distribution of these species in the different kinds of forests show that artificial coniferous stands have rebuilt a woodland avifauna which is not significantly different from that of spontaneous ones.

- Most of woodland bird communities of the Mont Ventoux are rich and well balanced as well in spontaneous as in artificial forests (table 4 ) but the Cedar stands are the richest and the most diversified, the reasons for which are discussed.

- The richness and Shannon's diversity are nicely correlated with the diversity of structure of the vegetation (fig. 1) in both spontaneous and artificial woods. 
- The more the structure of vegetation is complex, the more the bird community include species which are rare and/or severe in habitat selection (narrow niche breadth). Where the structure of vegetation is very simple as in coniferous monocultures, we find but few species that are very common anywhere and ubiquitous (large niche breadth).

- The coefficients of similitude between communities devised by the calculation of betadiversity are very high and do not allow to keep apart spontaneous stands from artificial ones. Eight forests among which 5 are spontaneous and 3 artificial make up a very homogeneous set linked together at the 0.75 level which is very high (fig. 3 . Community dendrogram).

It is concluded that both natural and artificial forests in the Mont Ventoux have rich and diversified bird communities, many of them having been rebuilt indirectly by man. Some mechanisms of habitat selection are discussed as well for the rich Cedar stands as for the relatively poor artificial Pinus uncinata woods.

The more forestry management increase the diversity of structure of vegetation, the more it will be successful for birds. It is pointed out that these results are concerned with the specific case of Mont Ventoux but cannot be generalized elsewhere in France without care.

\section{ZUSAMMENFASSUNG}

Fine ökologische Untersuchung an Vogelgemeinschaften an zwölf Stellen in spontanen und känstlichangeptlanzten Waldern des Ventoux-Massivs, eines Vorläufers der Alpen in Süd-Frankreich, hat Folgendes ergeben :

$5 \mathrm{r}$ Arten d.h. 6r p. 100 der Brutvogelarten wurden in den Wäldern dieses Massivs gezählt. Die Verteilung der Arten in den verschiedenen Waldgesellschaften zeigen, dass die. Aufforstungen eine Vogelgemeinschaft herausgebildet haben, die sich nicht grundliegend von der Vogelgemeinschaft der spontanen Wailder unterscheidet.

- Der reichste und vielseitigste Wald ist der Zedernwald. Im Durchschnitt, wie es Tabelle 4 zeigt, sind die Vogelgemeinschaften der spontanen und kunstlich angepflanzten Walder des Ventoux-Massivs reichhaltig und ausgeglichen.

- Die Reichhaltigkeit und die Diversitalt der Vogelgemeinschaften stehen eng in Beziehung mit der Diversität der Struktur der Vegetation (Abb. 1). Die Vögel reagieren in einem natiurlichen oder in einem künstlich angelegtem Wald ähnlich.

- Je reichhaltiger die Waldlandschaft, je mehr enthālt sie seltene und anspruchsvolle Arten. In einförmigen Nadelwaldern mit vereinfachten Strukturen, befinden sich nur allgemeinverbreitete Arten.

- Es ist nicht möglich eine Vogelgemeinschaft eines spontanes Waldes von einer eines künstlich aufgeforsteten Waldes zu unterscheiden. Acht Waldgesellschaften, darunter fünf spontane (Buchen-Tannen-, Kiefern-, Hakenkiefern-, Flaumeichen-, Kiefern-, und Flaumeichenwald) und drei künstliche (Schwarzfören-, Hakenkiefern- und Zedernwald) bilden hier ein gleichartiges Milieu.

Es wird darausgeschlossen, dass die spontanen und küntlichen Waldgesellschaften des Ventoux-Massivs reichhaltige und vielartige Vogelgemeinschaften aufweisen, einige unter ihnen wurden indirekt vom Menschen \& wiederhergestellt s. Einige Probleme der Raumbesetzung durch Vögel werden anhand der reichsten Waldgesellschaft (Zedernwald) und der ărmsten W. (kanstlich aufgeforsteter Hakenkiefernwald) erörtert. Die Schlussfolgerung lautet, dass der Erfolg einer Waldnutzung umso grösser ist, je vielfältiger die Strukturdiversität der Planzengemeinschaften ist.

Die grundsätzliche Verschiedenheit zwischen einem Nutzwald und einem Schutzwald wird unterstrichen, insofern sich die hier dargestellten Schlussfolgerungen nur auf das spezifische Beispiel des Ventoux-Massivs beziehen,

\section{RÉFÉRENCES BIBLIOGRAPHUUES}

Arrtent J., rg72. Forét cornmunale de Bédoin. Doc. polycopié, O. N. F. Avignon, 8 p.

Baraero M., du Merle P., Quzzel. P., 1976. Les peuplements sylvatiques du Mont Ventoux (Vaucluse). Documents phylosnciologiques, 15-18, I-I4-

Barkero M., Quezes. P., 1975. Végétation culminale du Mont Ventoux, sa signification dans une interprétation phytogéographique des Préalpes méridionales. Ecologia Mediterranca, 1, 1-33.

Annales des Sciences forestières. - 1976 . 
Btoxpes. J., 1975. C'analyse des peuplements d’oisesaux, éhùment d'un diagnostic écologigue. I. - La méthode des Echantillonnages fréquentids progressifs (B. F, P.). La Terre et la Vie, 1975, 4, 533.589.

Blondel J., Ferky C., Frocnor R., 1973. Avifaune et végétation, essai d'analyse de la diversité. Aluade, $41,63-84$.

Dacier Ph., 1975. Aspects structurels de la végétation herbacée de I'aire P., B. 1. du Solling (Allemagne). C.N.R.S.C.E.P.E., Doc. n० 61 .

Eyaent M. C., I972. Étude de Pavifaune nicheuse d'un massif forestier à eurésinement croisant. C. R. Acad. Sci, Paris, 275, $1815-1818$.

Fеких C., 1959. Ritudes quantitatives sur les oiseaux forestiers. Revae forcstione francaice, 3, $173-185$.

FERreY C., Frochot B., rg7o. L'avifaune nidificatrice d'une forèt de Chùnes pédonculés en Baurgogne : étude de deux successions Ecologiques. La Terre at ha Vie, 24, 153-250.

Perse C., Frochot B., r974. L'influence du traitement forestier sur les oiseaux. In : Prssos P. Ecologie forestiere, Gauthiers-Villars, Paris, $3009-326$,

Frochot B., 1975. Influence de lexploitation forestiere sur lis reproduction des oiseaux. Bull. Soc. Zool. de France, 100, 77-84.

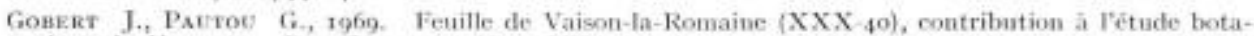
nique du Ventoux. Doc, Carfe vég. Alpes, V11, 145 - 194.

Mantuss C. F., 1838. Essai sur la topographie botanicjue du Mont Ventoux, en Provence. Ann. Sa. Nat, , $2^{n}$ série, to.

Maury P., 1960, Le reboisement de la forcit de Bédoin et son enseignement. Ann. École Nat. E. at F., $17,119-149$.

Nígre M., 1931. Les reboisentents du Massif de T'Aigoual, Bull. Soc, E2, Sci, nat, Nimes, $135 \mathrm{p}$,

Nolrfar.1sp. A., 1964. Conséquences écologiques de la monoculture des Coniferes dans la zone des Feuillus de I'Europe tempéréc. Rapp. Conseil de l'Europe, 36 p.

Pnctex J., 1967. Die Bestandichte der Voselpopulationen einer sckundaren Geselischaft mit uberwiegender Fichte. Zool. Listy, 16, $173-182$.

Toru J-, 1970-1972, Historique du Cedre sur le Mtont Ventoux. Eull. Sac. El. Sci, nat, Vaurdase, 5 t-75.

Witliasion K., r97o, Birds and modern forestry. Quart. J. For., 64, 345-355.

Wiflinstson K., 1972. The conservation of Bird life in the new coniferous forests, Forestry, 45, 87.100, Yapp W. B, 1962. Birds and woods. Oxford Univ. Press.

\section{ANNEXE}

(nom latin des espèces citées dans le texte)

Êpervier

Autour

Circaëte

Hibou grand-duc

Pigeon ramier

Tourterelle

Coucou gris

Pic-vert

Pic-épeiche

Pic-noir

Alouette lulu

Hirondelle de rochers

Hirondelle de cheminée

Corneille noire

Pie

Geai
Accipiter wisus. Accipiter gentilis. Circaetus gallicus.

Bubo bubo.

Columba palumbus.

Streptopelia turtur.

Cuculus canorus.

Picus viridis.

D.ndrocopos major.

Dryocopus martius.

Lullula arborea.

Riparia rupestris.

Hirundo rustica.

Corvus corone.

Pica pica.

Garrulus glandarius. 
Mésange charbonnière

Mésange bleue

Mésange noire

Mésange huppée

Mésange nonnette

Mésange à longue queue

Sittelle

Grimpereau des jardins

Troglodyte

Grive draine

Grive musicienne

Merle

Rouge queue à front blanc

Rossignol

Rouge-gorge

Traquet oreillard

Fauvette à tête noire

Fauvette grisette

Fauvette orphée

Fauvette mélanocéphale

Fauvette passerinette

Fauvette pitchou

Pouillot véloce

Pouillot de bonelli

Roitelet huppé

Roitelet triple bandeau

Accenteur mouchet

Pipit des arbres

Verdier

Chardonneret

Linotte

Venturon

Serin cini

Bouvreuil

Beccroisé

Pinson

Bruant zizi

Bruant fou

Moineau domestique

Moineau friquet
Parus major.

Parus casruleus.

Parus ater.

Parus cristalus.

Parus palustris.

Aegilhalos caudatus.

Sitta europaea.

Certhia brachydactyla

Troglodytes troglodytes.

Turdus viscivorus.

Turdus philomelos.

Turdus merula.

Phoenicurus phoenicurtrs.

Luscinia megarhynchos.

Erithacus rubecula.

Oenanthe hispanica.

Sylvia atricapilla.

Sylvia communis.

Sylvia hortensis.

Sylvia melanocephala.

Sylvia cantillans.

Sylvia undata.

Phylloscopus collybita.

Phylloscopus bonelli.

Regulus regulus.

Reguius ignicapillus.

Prunella modularis.

Anthus trivialis.

Carduelis chloris.

Carduclis carduelis.

Carduelis cannabina.

Carduelis citrinella.

Serinus canaria.

Pyrrhula pyrrhula.

Loxia curvirostra.

Fringilla coelebs.

Emberiza cirlus.

Emberiza cia.

Passer domesticus.

Passer montanus. 